\title{
First application of the Oslo method in inverse kinematics
}

\author{
Nuclear level densities and $\gamma$-ray strength functions of ${ }^{87} \mathrm{Kr}$
}

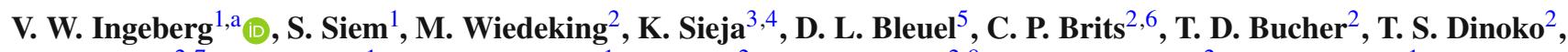
J. L. Easton ${ }^{2,7}$, A. Görgen ${ }^{1}$, M. Guttormsen ${ }^{1}$, P. Jones ${ }^{2}$, B. V. Kheswa ${ }^{2,8}$, N. A. Khumalo ${ }^{2}$, A. C. Larsen ${ }^{1}$, E. A. Lawrie $^{2}$, J. J. Lawrie ${ }^{2}$, S. N. T. Majola ${ }^{2,8,9}$, K. L. Malatji ${ }^{2,6}$, L. Makhathini ${ }^{2,6}$, B. Maqabuka ${ }^{2,7}$, D. Negi ${ }^{2}$, S. P. Noncolela ${ }^{2,7}$, P. Papka ${ }^{2,6}$, E. Sahin ${ }^{1}$, R. Schwengner ${ }^{10}$, G. M. Tveten ${ }^{1}$, F. Zeiser ${ }^{1}$, B. R. Zikhali ${ }^{2,9}$

${ }^{1}$ Department of Physics, University of Oslo, 0316 Oslo, Norway

2 iThemba LABS, P.O. Box 722, Somerset West 7129, South Africa

${ }^{3}$ Université de Strasbourg, IPHC, 23 rue du Loess, 67037 Strasbourg, France

${ }^{4}$ CNRS, UMR7178, 67037 Strasbourg, France

${ }^{5}$ Lawrence Livermore National Laboratory, 7000 East Avenue, Livermore, CA 94550-9234, USA

${ }^{6}$ Department of Physics, Stellenbosch University, Private Bag X1, Matieland 7602, South Africa

${ }^{7}$ Department of Physics, University of the Western Cape, P/B X17, Bellville 7535, South Africa

${ }^{8}$ Department of Physics, University of Johannesburg, P.O. Box 524, Auckland Park 2006, South Africa

${ }^{9}$ Department of Physics, University of Zululand, Private Bag X1001, KwaDlangezwa 3886, South Africa

${ }^{10}$ Institut für Strahlenphysik, Helmholtz-Zentrum Dresden-Rossendorf, 01328 Dresden, Germany

Received: 12 July 2019 / Accepted: 14 January 2020 / Published online: 20 February 2020

(C) The Author(s) 2020

Communicated by Ari Jokinen

\begin{abstract}
The $\gamma$-ray strength function $(\gamma \mathrm{SF})$ and nuclear level density (NLD) have been extracted for the first time from inverse kinematic reactions with the Oslo method. This novel technique allows measurements of these properties across a wide range of previously inaccessible nuclei. Proton $-\gamma$ coincidence events from the $\mathrm{d}\left({ }^{86} \mathrm{Kr}, \mathrm{p} \gamma\right){ }^{87} \mathrm{Kr}$ reaction were measured at iThemba LABS and the $\gamma \mathrm{SF}$ and $\mathrm{NLD}$ in ${ }^{87} \mathrm{Kr}$ was obtained. The low-energy region of the $\gamma \mathrm{SF}$ is compared to shell-model calculations, which suggest this region to be dominated by M1 strength. The $\gamma \mathrm{SF}$ and NLD are used as input parameters to Hauser-Feshbach calculations to constrain (n, $\gamma$ ) cross sections of nuclei using the TALYS reaction code. These results are compared to ${ }^{86} \mathrm{Kr}(n, \gamma)$ data from direct measurements.
\end{abstract}

\section{Introduction}

The nuclear level density (NLD) and the $\gamma$-ray strength function $(\gamma \mathrm{SF})$ are fundamental properties of the nucleus. The NLD was introduced by Bethe soon after the composition of nuclei was firmly established [1]. When excitation energy in a nucleus increases towards the particle separation energy, the NLD increases rapidly, creating a region referred to as the quasi-continuum. The ability of atomic nuclei to emit and

a e-mail: vetlewi@fys.uio.no (corresponding author) absorb photons in the quasi-continuum is determined by the $\gamma \mathrm{SF}$ [2]. It is a measure of the average reduced $\gamma$-ray decay probability and reveals essential information about the electromagnetic response and therefore the nuclear structure of the nucleus.

With their significant applicability to astrophysical element formation via capture processes [3-6], NLDs and $\gamma$ SFs have received increased experimental and theoretical attention [7]. They are also relevant to the design of existing and future nuclear power reactors, where reactor simulations depend on many evaluated nuclear reactions $[8,9]$. The importance of NLDs and $\gamma$ SFs is increasingly being recognized and a reference database for $\gamma$ SFs has been established [10]. Nonetheless, challenges remain and nuclear physics properties, such as the NLD and $\gamma \mathrm{SF}$, remain a main source of uncertainty in cross-section calculations. This is either due to the complete lack of experimental data or the associated large experimental uncertainties.

The situation can be improved through accurate experimental neutron capture cross sections, or indirectly by measuring NLD and $\gamma \mathrm{SF}$ data. One experimental approach, the Oslo method [11], has been extensively used to measure the NLD and $\gamma$ SF from particle- $\gamma$ coincident data. NLDs and $\gamma$ SFs obtained with the Oslo method have been shown to provide reliable neutron capture cross sections $[12,13]$ and proton capture cross sections [14]. In recent years, the Oslo 
method has been extended to extract the $\gamma$ SF and NLD following $\beta$ decay [15]. Using $\gamma$ SFs and NLDs to determine capture cross sections has several advantages since these properties can be obtained for any nucleus that can be populated in a reaction from which the excitation energy can be experimentally determined. Although the Oslo and $\beta$-Oslo methods provide access to a vast range of stable and radioactive nuclei some species remain inaccessible. Many more nuclei become accessible by using inverse kinematic reactions, from radioactive species to several stable isotopes for which the manufacture of targets is problematic due to their chemical or physical properties.

In this Letter we report on the first application to measure the NLD and $\gamma \mathrm{SF}$ with the Oslo method following an inverse kinematic reaction. This work lays the foundation of new opportunities to study statistical properties of nuclei, which were previously inaccessible, at stable and radioactive ion beam facilities. The results from the $\mathrm{d}\left({ }^{86} \mathrm{Kr}, \mathrm{p}\right){ }^{87} \mathrm{Kr}$ reaction exhibit a low-energy enhancement of the $\gamma \mathrm{SF}$ in ${ }^{87} \mathrm{Kr}$, which is discussed in the context of shell-model calculations. The ${ }^{86} \mathrm{Kr}(\mathrm{n}, \gamma)$ cross section is obtained from the TALYS reaction code [16] and compared to previous direct measurements to test the robustness of the experimental method.

\section{Experiment}

The experiment was performed with a $300 \mathrm{MeV}{ }^{86} \mathrm{Kr}$ beam from the Separated Sector Cyclotron facility at iThemba LABS. Polyethylene targets with $99 \%$ deuteron enrichment were bombarded with a beam intensity of $\approx 0.1 \mathrm{pnA}$ for $80 \mathrm{hrs}$. Several deuterated polyethylene targets, ranging in thicknesses from 110 to $550 \mu \mathrm{g} / \mathrm{cm}^{2}$, were used. Accounting for the target thicknesses the center-of-mass (CM) energy was 6.44(40) MeV. The reactions were identified through the detection of light charged particles in two silicon $\Delta E-E$ telescopes covering scattering angles between $24^{\circ}$ and $67^{\circ}$ relative to the beam direction (corresponding to $\mathrm{CM}$-angles $38^{\circ}-121^{\circ}$ ). The $E$ detectors were $1 \mathrm{~mm}$ thick while the $\Delta E$ detectors were 0.3 and $0.5 \mathrm{~mm}$ thick. The dimensions of the W1-type double-sided silicon strip detectors [17] were $4.8 \times 4.8 \mathrm{~cm}$ and they consisted of 16 parallel and perpendicular strips $3 \mathrm{~mm}$ wide with an opening angle of $\approx 1.5^{\circ}$ for each pixel. Suppression of $\delta$ electrons was achieved by an aluminum foil of $4.1 \mathrm{mg} / \mathrm{cm}^{2}$ areal density which was placed in front of the $\Delta E$ detectors. The $\gamma$-rays were measured with the AFRODITE array [18], which at the time of the experiment consisted of eight collimated and Compton suppressed high-purity germanium CLOVER-type detectors. Two noncollimated $\mathrm{LaBr}_{3}: \mathrm{Ce}$ detectors $\left(3.5^{\prime \prime} \times 8^{\prime \prime}\right)$ were coupled to the AFRODITE array and mounted $24 \mathrm{~cm}$ from the target at $45^{\circ}$. The detectors were calibrated using standard ${ }^{152} \mathrm{Eu}$ and ${ }^{56} \mathrm{Co}$ sources. The detector signals were processed by XIA digital electronics in time-stamped list mode with each channel self-triggered.

From the time-stamped list mode data, entries were selected based on their time-stamps being within a window of $\pm 1850 \mathrm{~ns}$ in an $E$-detector entry. The ratio of energy deposited in the $\Delta E$ - to the $E$-detector is used to determine the outgoing reaction channels. The selection of proton$\gamma$ events was made with an $80 \mathrm{~ns}$ wide time-gate on the prompt time peak. Contributions from uncorrelated events were subtracted from the data by placing off-prompt time gates of equal length. This leads to approximately $100 \mathrm{k}$ proton $-\gamma$ events in both $\mathrm{LaBr}_{3}$ :Ce and CLOVER matrices. In this letter only the data from the $\mathrm{LaBr}_{3}: \mathrm{Ce}$ detectors are included, although data from the CLOVER detectors yield similar results. Kinematic corrections due to the reaction $Q$ value, recoil energy of ${ }^{87} \mathrm{Kr}$, and the energy losses of the protons in the target and aluminum foils were applied to determine the excitation energy of the populated states, with the a resulting FWHM for excitation energy of $\approx 1 \mathrm{MeV}$. The $\gamma$-rays in coincidence with protons were Doppler corrected by assuming the residual ${ }^{87} \mathrm{Kr}$ nucleus not being deflected from the beam axis and has a constant velocity of $8.5 \%$ of $\mathrm{c}$. Due to these assumptions the error in deflection angle is less than $1.3^{\circ}$ while the error in velocity is less than $0.4 \%$ of $\mathrm{c}$. These errors are negligible as the major contributor to errors in the Doppler correction is the $17^{\circ}$ opening angle of the $\mathrm{LaBr} 3: \mathrm{Ce}$ detectors. Background from ${ }^{86} \mathrm{Kr}+{ }^{12} \mathrm{C}$ fusion evaporation events has been simulated with PACE4 [19] and was found to have a very low proton yield $(<4 \%)$ with proton energies outside the energy range considered in the analysis. This matrix is unfolded [20] with response functions of the detectors extracted from a Geant4 [21] simulation of the $\mathrm{LaBr}_{3}: \mathrm{Ce}$ detectors. An iterative subtraction method, known as the first-generation method [22], is applied to the unfolded $\gamma$ ray spectra, revealing the distribution of primary $\gamma$-rays in each excitation bin ( $256 \mathrm{keV}$ bin width for both the $E_{x}$ and $E_{\gamma}$ axes).

The NLD $\rho\left(E_{x}\right)$ at excitation energy $E_{x}$ and $\gamma$-ray transmission coefficient, $\mathcal{T}\left(E_{\gamma}\right)$, are related to the primary $\gamma$-ray spectrum by [11]

$P\left(E_{x}, E_{\gamma}\right) \propto \rho\left(E_{x}-E_{\gamma}\right) \mathcal{T}\left(E_{\gamma}\right)$,

and are extracted with a $\chi^{2}$-method [11] giving the unique solution of the functional shape of the NLD and $\mathcal{T}\left(E_{\gamma}\right)$. These are normalized to known experimental data to retrieve the correct slope and absolute value. The extraction has been performed within the limits $3.2<E_{x}<5.2 \mathrm{MeV}$ and $E_{\gamma}>$ $1.7 \mathrm{MeV}$ of the primary $\gamma$-ray matrix where the level density is sufficiently high for statistical decay to be dominant. 


\section{Normalization}

From the primary $\gamma$-ray spectrum the $\operatorname{NLD} \widetilde{\rho}\left(E_{x}\right)$ and $\gamma$ transmission coefficient $\widetilde{\mathcal{T}}\left(E_{\gamma}\right)$ are extracted. These are related to the physical solution by the following transformation [11]:

$$
\begin{gathered}
\rho\left(E_{x}\right)=A \widetilde{\rho}\left(E_{x}\right) e^{\alpha E_{x}} \\
\mathcal{T}\left(E_{\gamma}\right)=B \widetilde{\mathcal{T}}\left(E_{\gamma}\right) e^{\alpha E_{\gamma}},
\end{gathered}
$$

where $A$ and $B$ are the absolute values for the level density and the transmission coefficient, respectively, and $\alpha$ is the common slope parameter.

For the level density, the slope and absolute value are determined by a fit to the level density found from the known discrete levels [23] at low-excitation energy and the level density at the neutron separation energy $\left(S_{n}=5.5 \mathrm{MeV}\right)$. The level density of $J=1 / 2$ levels at $S_{n}$ is determined from the average resonance spacing of s-wave resonances $\left(D_{0}\right)$ and p-wave resonances $\left(D_{1}(J=1 / 2)\right)$ by

$\rho(S n, J=1 / 2)=\frac{1}{D_{0}}+\frac{1}{D_{1}(J=1 / 2)}$,

with the spacing parameters are taken from [24]. The full level density at $S_{n}$ is determined by

$\rho\left(S_{n}\right)=\rho\left(S_{n}, J=1 / 2\right) / g(S n, J=1 / 2)$,

where $\mathrm{g}$ is the spin distribution [25]

$g(E, J)=\frac{2 J+1}{2 \sigma^{2}(E)} e^{-(J+1 / 2)^{2} / 2 \sigma^{2}(E)}$.

The spin cutoff parameter $\sigma(E)$ is modeled with the following energy dependence [12]:

$\sigma^{2}(E)=\sigma_{d}^{2}+\frac{E-E_{d}}{S_{n}-E_{d}}\left(\sigma^{2}\left(S_{n}\right)-\sigma_{d}^{2}\right)$,

where $E_{d}$ is the excitation energy below which the spin cutoff parameter $\sigma=\sigma_{d}$ is a constant. The spin cutoff parameter $\sigma_{d}$ at $E_{d} \leq 2.4 \mathrm{MeV}$ is estimated to be $1.75(26)$, based on the spin assignment of the known levels, while the cutoff parameter at the neutron separation energy $\sigma\left(S_{n}\right)$ is estimated to be $3.95(60)$, based on the predictions of the spin cutoff models of Refs. [26-28]. The shape of the spin distribution predicted by the Hartree-Fock-Bogoliubov plus combinatorial model [29] has also been considered and found to be in agreement. Based on the estimated uncertainties of $\sigma(E)$ and the experimental uncertainties of the resonance spacing, the total NLD at $S_{n}$ is found to be $1472(427) \mathrm{MeV}^{-1}$.

The level density extracted with the Oslo method extracted extends up to $3.7 \mathrm{MeV}$ and an interpolation between the Oslo method data and the neutron separation energy has to be done. This interpolation uses the constant temperature (CT) shape [30]

$\rho_{\mathrm{CT}}(E)=\frac{1}{T} e^{\frac{E-E_{0}}{T}}$,

with shift parameter $E_{0}=S_{n}-T \ln \left(T \rho\left(S_{n}\right)\right)$ to ensure that the interpolation matches the experimental known $\rho\left(S_{n}\right)$. The optimum temperature parameter $T$ in the interpolation, as well as the normalization parameters $A$ and $\alpha$, are determined through a least-squares fit between the level density extracted in the Oslo method and the discrete levels for energies below $E_{x}=2.4 \mathrm{MeV}$ and the CT interpolation above.

Since the reaction is sub-Coulomb barrier the primary reaction channel will be neutron capture following inelastic deuteron breakup in the Coulomb field of the ${ }^{86} \mathrm{Kr}$ projectile and $1 / 2$ states are assumed to be strongly favored in the initial population, and has to be accounted for. Since the resulting normalized level density found with the Oslo method will correspond to the level density of $1 / 2$ and $3 / 2$ levels, the total level density is recovered by dividing by $g\left(E_{x}, 1 / 2\right)+g\left(E_{x}, 3 / 2\right)$.

The same normalization procedure has been repeated, but with an interpolation with a shape matching that of the BackShifted Fermi-gas model [27,31] with the difference in the resulting normalization included in the error bars. All errors due to systematical and statistical effects of the Oslo method [32], together with those related to the normalization process have been propagated to give the level density with error bars shown in Fig. 1.

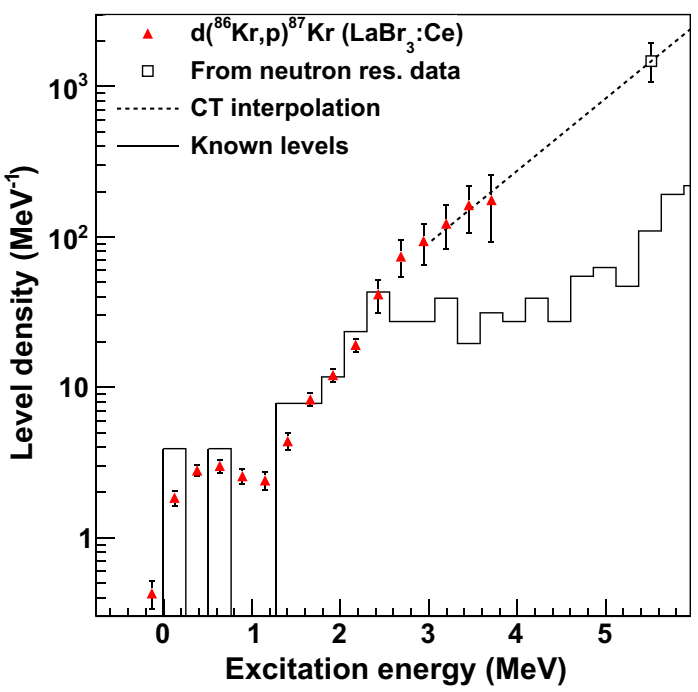

Fig. 1 Normalized ${ }^{87} \mathrm{Kr}$ nuclear level densities for $\mathrm{LaBr}_{3}$ :Ce (red circles) detectors. The black line shows the known levels while the open square is the level density at the neutron separation energy. The dashed line is the constant temperature interpolation. The error bars represent the upper and lower uncertainty limit due to all known statistical and systematic effects 


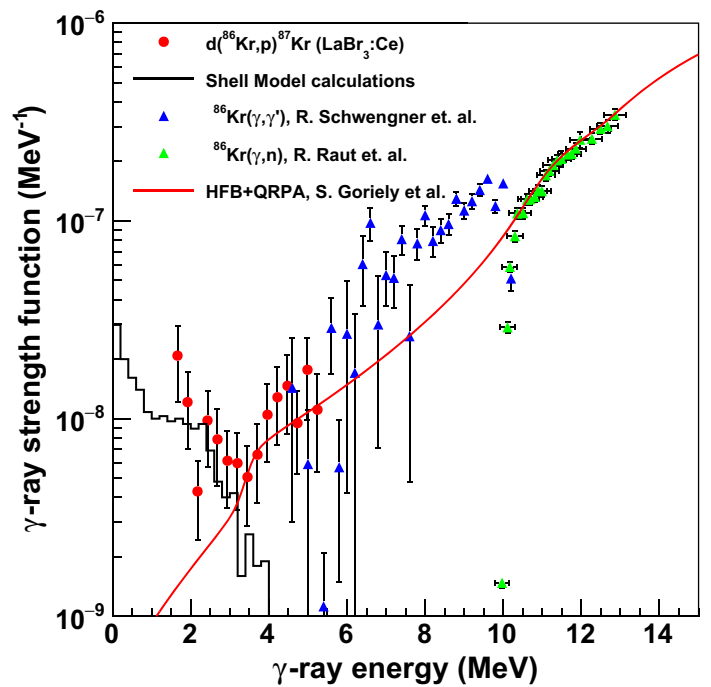

Fig. $2 \gamma$-ray strength function of ${ }^{87} \mathrm{Kr}$ (red circles) compared with the $\gamma$-ray strength function of ${ }^{86} \mathrm{Kr}$ extracted from ${ }^{86} \mathrm{Kr}\left(\gamma, \gamma^{\prime}\right)$ (blue triangles) [35] and ${ }^{86} \operatorname{Kr}(\gamma, \mathrm{n})$ (green squares) [36]. The solid black line are results from Shell Model calculations with a ${ }^{78} \mathrm{Ni}$ core (see Sect. 5 for details), while the red line is the microscopic HFB+QRPA prediction [37] for the E1 strength. The error bars include all known statistical and systematic errors

Table 1 Experimental values and parameters used in the normalization. The spin cutoff at $S_{n} \sigma(S n)$ is an average of the models presented in [26-29] while $\sigma_{d}$ is estimated from discrete states with known spin. The level density of $1 / 2$ levels at $S_{n}$ are found using Eq. (4) and the total level density at $S_{n}$ with Eq. (5). The temperature $T$ is determined from a least-squares fit to data points in the range $2.4<E_{x}<3.7 \mathrm{MeV}$

\begin{tabular}{ll}
\hline$D_{0}$ & $26.2(21) \mathrm{keV} \mathrm{[24]}$ \\
$D_{1}(J=1 / 2)$ & $18.8(14) \mathrm{keV}[24]$ \\
$\sigma\left(S_{n}\right)$ & $3.95(60)$ \\
$\sigma_{d}$ & $1.75(26)$ \\
$\rho\left(S_{n}, 1 / 2\right)$ & $91(5) \mathrm{MeV}^{-1}$ \\
$\rho\left(S_{n}\right)$ & $1472(427) \mathrm{MeV}^{-1}$ \\
$\left\langle\Gamma_{\gamma 0}\right\rangle$ & $0.25(10) \mathrm{eV}[33]_{T}$ \\
\hline
\end{tabular}

The absolute value of the transmission coefficients are normalized to the average radiative width of s-wave resonances $\left\langle\Gamma_{\gamma 0}\right\rangle$ in a process detailed in [34], and converted to $\gamma$ SF by $f\left(E_{\gamma}\right)=\mathcal{T}\left(E_{\gamma}\right) /\left(2 \pi E_{\gamma}^{3}\right)$. The value of $\left\langle\Gamma_{\gamma 0}\right\rangle$ is estimated to be $0.25(10) \mathrm{eV}$ based on the measured $\Gamma_{\gamma}$ of s-wave resonances of [33]. The resulting $\gamma \mathrm{SF}$ with all errors propagated are shown in Fig. 2. All experimental values and parameters used in the normalization process are listed in Table 1.

\section{Nuclear level densities and $\gamma$-ray strength functions}

The normalized NLD is shown in Fig. 1 and is in excellent agreement with the constant temperature level density and matches well with the known discrete states at lower excitation energies. The normalized $\gamma \mathrm{SF}$ is shown in Fig. 2 and is consistent with $\gamma$ SFs from ${ }^{86} \operatorname{Kr}\left(\gamma, \gamma^{\prime}\right)$ [35] and ${ }^{86} \operatorname{Kr}(\gamma, \mathrm{n})$ [36], with the enhancement seen in the $\left(\gamma, \gamma^{\prime}\right)$ data between 6 and $8 \mathrm{MeV}$ caused by a Pygmy resonance [35]. A drop in the $\gamma \mathrm{SFs}$ at $\sim 2.1 \mathrm{MeV}$ is caused by the $2123-\mathrm{keV}$ state in ${ }^{87} \mathrm{Kr}$, which is strongly populated in the reaction, but less through feeding from the quasi-continuum. This causes the first-generation method to over-subtract in the higher excitation-energy bins, causing an artificial drop in the $\gamma \mathrm{SF}$. This effect has previously been discussed [32]. At low energies we observe a large enhancement in the $\gamma \mathrm{SF}$, similar to what has been observed in several other nuclei [38-44]. Although the upbend has been independently confirmed [45], little is known of the origin of this feature, except that it is dominated by dipole radiation [46-48] and that it can have large effects on neutron capture cross sections [49].

\section{Shell-model calculations}

Calculations within the shell-model framework predicts the upbend due to M1 transitions [50]. In this work, largescale shell-model calculations of the M1 component of the $\gamma \mathrm{SF}$ were performed in the model space outside the ${ }^{78} \mathrm{Ni}$ core, containing $f_{5 / 2} p_{3 / 2} p_{1 / 2} g_{9 / 2}$-proton and $d_{5 / 2} s_{1 / 2}$ $d_{3 / 2} g_{7 / 2} h_{11 / 2}$-neutron orbitals. The effective interaction employed here is described e.g. in Refs. [51,52]. The diagonalization of the Hamiltonian matrix in the full configuration space was achieved using the Strasbourg shell-model code NATHAN [53]. The spin part of the magnetic operator was quenched by a common factor of 0.75 [53]. We computed this way up to 60 states of each spin between $1 / 2$ and $15 / 2$ for both parities. This leads to a total of around $8 \cdot 10^{4}$ M1 matrix elements, among which 14,822 connect states located in the energy range $E_{x}=3.4-$ $5.4 \mathrm{MeV}$, as considered in the experiment. To obtain the average strength per energy interval, $\langle B(M 1)\rangle$, the total transition strength was accumulated in $200 \mathrm{keV}$ bins and divided by the number of transitions within these bins. The $\gamma \mathrm{SF}$ was obtained from the relation $f_{M 1}\left(E_{\gamma}, E_{i}, J_{i}, \pi\right)=$ $16 \pi / 9(\hbar c)^{-3}\left\langle B(M 1)\left(E_{\gamma}, E_{i}, J_{i}, \pi\right)\right\rangle \rho\left(E_{i}, J_{i}, \pi\right)$, where $\rho_{i}\left(E_{i}, J_{i}, \pi\right)$ is the partial level density at the energy of the initial state $\left(E_{i}\right)$. The $\gamma \mathrm{SF}$, shown in Fig. 2, is an average of the $f_{M 1} \mathrm{~s}$ evaluated for each spin/parity separately. The shape of the shell-model $\gamma \mathrm{SF}$ is consistent with experimental data up to $\sim 3 \mathrm{MeV}$. Since the model space does not contain all spin-orbit partners (i.e., $v g_{9 / 2}$ and $\pi f_{7 / 2}$ orbits) the strength above $4 \mathrm{MeV}$, due to the spin-flip transitions, cannot be accounted for. However, the theoretical $\gamma \mathrm{SF}$ exhibits significant strength at $E_{\gamma}=0$, as in the previous shell-model calculations in this mass region [50]. The largest $B(M 1)$ contributions at low $\gamma$-ray energies in ${ }^{87} \mathrm{Kr}$ 
are related to transitions between close-lying negative-parity states with $\nu d_{5 / 2} \otimes \pi f_{5 / 2}^{-1} g_{9 / 2}^{1}$ and $\nu d_{5 / 2} \otimes \pi p_{3 / 2}^{-1} g_{9 / 2}^{1}$ components. The magnitude of the theoretical M1 strength is in good agreement with the data as measured in the experiment; however, we cannot exclude an additional contribution from E1 strength. Recent experimental results in ${ }^{56} \mathrm{Fe}$ [48] could suggest a mixture of M1 and E1 radiation in the enhancement region and the addition of a non-zero E1 component without an upbend towards $E_{\gamma} \rightarrow 0 \mathrm{MeV}$ is predicted from shell-model calculations [54]. Including the E1 strength calculations from the Hartree-Fock-Bogolyubov + QRPA (HFB+QRPA) model by [37] we observe an overall good agreement between theoretical predictions and experimental results.

\section{Neutron capture cross sections}

In a statistical framework the ${ }^{86} \mathrm{Kr}(\mathrm{n}, \gamma)$ cross section can be determined from the NLD, $\gamma \mathrm{SF}$ and a suitable neutron optical model potential (nOMP) for ${ }^{87} \mathrm{Kr}$. Phenomenological nOMPs e.g. from Ref. [55] are observed to give good agreement with the total cross section for nuclei close to the valley of stability. We performed Hauser-Feshbach (HF) [56] calculations with the TALYS ${ }^{1}$ code [16], and the optical model potential of Ref. [55]. A semi-microscopic optical model [57] was also tested, and gave virtually the same results. Pre-equilibrium reactions were also taken into account. Tabulated experimental NLD are passed to TALYS, with NLD at energies above $3.7 \mathrm{MeV}$ generated from the CT interpolation. Up to $2.3 \mathrm{MeV}$ the known discrete levels are used. E1 and M1 strength are tabulated from the experimental $\gamma \mathrm{SF}$ (1.6 $\leq E_{\gamma} \leq 5.2 \mathrm{MeV}, 2.1 \mathrm{MeV}$ data point excluded) with the strength outside the experimental region tabulated from the microscopic HFB + QRPA calculations of [37] for the E1 strength and the strength found in the SM calculations plus a standard Lorentzian for the M1 spin flip with the default TALYS parameterization. Decomposition of the experimental $\gamma \mathrm{SF}$ are done by subtracting the SM + spin-flip strength and assuming the residual being E1.

The capture cross section for astrophysical relevant neutron energies are proportional to the integrated product of the NLD and $\gamma \mathrm{SF}$ at all energies from the ground state to the neutron separation energy and are the region experimentally determined. The resulting neutron capture cross section are shown in Fig. 3. The input parameters have been varied in accordance with the statistical and systematic uncertainties to produce the red-hashed error-band. We observe an overall good agreement with direct measurements by Bhike et al. [58] and a decent agreement at higher energies with measurements of Walter et al. [59], while somewhat high compared

1 Version 1.9.

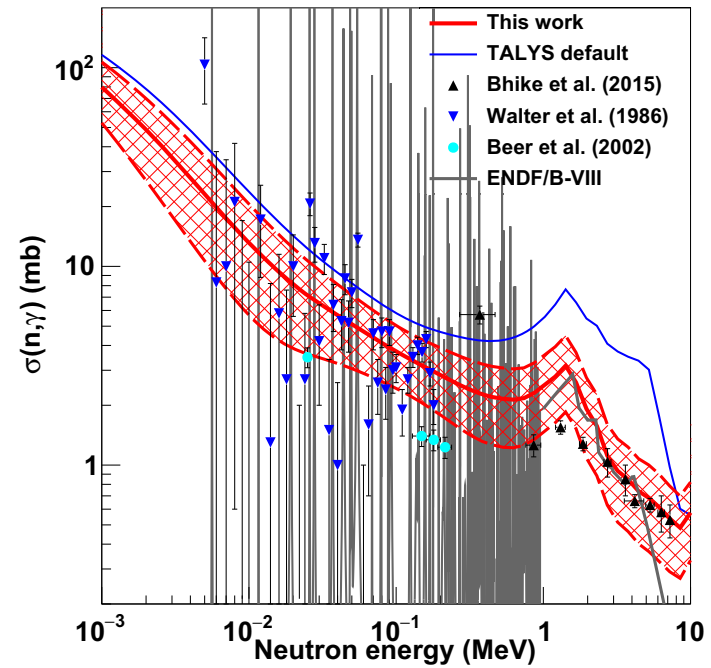

Fig. $3{ }^{86} \operatorname{Kr}(n, \gamma)$ cross sections. The red-hashed area represents the total uncertainty based on both systematical and statistic errors. The gray and blue lines are from the evaluation of ENDF/B-VII.1 [8] and the TALYS default input, respectively, and is provided for comparison. The black triangles shows the direct measurements of Bhike et al. [58], the blue upside-down triangles are results from time-of-flight measurements of Walter et al. [59] and the turquoise circles are the results from the activation measurements of Beer et al. [60]

with the activation results of Beer et al. [60]. The Maxwellian average (MACS) at the typical s-process temperature of 30 $\mathrm{keV}$ is found to be 7.2(36) mb, which is higher than the evaluated value of 3.4(3) mb found in KaDoNis [61]. This discrepancy can be explained by the fact that HF calculations will give results that overestimate the MACS for low temperatures when the level density is low [62]. A possible resolution could be to use Monte Carlo simulations to generate statistical resonances from average nuclear properties as proposed in $[63,64]$.

\section{Conclusion}

We have presented a novel method for obtaining $\gamma \mathrm{SF}$ and NLD using inverse kinematic reactions, which opens opportunities to study a wide range of stable and radioactive nuclei. The $d\left({ }^{86} \mathrm{Kr}, p \gamma\right)$ reaction was used to measure the NLD and $\gamma \mathrm{SF}$ in ${ }^{87} \mathrm{Kr}$. The low-energy part of the $\gamma \mathrm{SF}$ is found to exhibit an enhancement. Shell-model calculations were performed and suggest that the enhancement is predominantly due to low-energy M1 transitions in ${ }^{87} \mathrm{Kr}$.

The $\gamma \mathrm{SF}$ and NLD measurements in ${ }^{87} \mathrm{Kr}$ were used to calculate $(n, \gamma)$ cross sections, which are in good agreement with those from direct measurements, and give confidence in the approach using inverse kinematic reactions. This is consistent with the findings of previous work with the Oslo method and is particularly interesting since direct measure- 
ment of neutron capture cross sections over a wide range of incident neutron energies is very challenging. It is clear that $\gamma$ SFs and NLDs provide a viable alternative to obtain reliable capture cross sections.

With inverse kinematics, new regions of the nuclear chart become accessible to experiments, which also brings about new challenges. For exotic nuclei, neutron resonance data are not known and the normalizing procedure needs to be revised. One possibility is that the slope of the $\gamma \mathrm{SF}$, and thereby also the slope of the NLD, could be constrained using a technique where the ratio of populated discrete states from the quasicontinuum is used to determine the shape of the $\gamma \mathrm{SF}[45,65]$, leaving the absolute value of the NLD to be determined by the known discrete levels. Unfortunately, this still does not determine the absolute value of the $\gamma$ SF. However, reasonable estimates of the absolute value may be obtained from systematics of the $\left\langle\Gamma_{\gamma 0}\right\rangle$.

Measuring statistical properties of nuclei from inverse kinematic reactions provides a novel and complementary foundation for exploring the limitations of the current models of statistical behavior in the nucleus. It will allow for further constraining the uncertainties in models which are used in nuclear astrophysics and reactor physics.

Acknowledgements Open Access funding provided by the University of Oslo \& Oslo University Hospital. The authors would like to thank iThemba LABS operations for stable running conditions and John Greene (Argonne National Lab.) for providing excellent targets. This work is based on research supported by the Research Council of Norway under project Grants no. 222287, 262952 (G. M. T.), 263030 (V. W. I, S. S., A. G., F. Z.) and 240104 (E.S), by the National Research Foundation of South Africa under grant no 118846, and the U.S. Department of Energy by Lawrence Livermore National Laboratory under Contract DE-AC52-07NA27344. A. C. L. gratefully acknowledges funding through ERC-STG-2014 Grant Agreement no. 637686, and support from the ChETEC Cost Action (CA16117) supported by COST. This work was performed within the IAEA CRP on "Updating the Photonuclear data Library and generating a Reference Database for Photon Strength Functions" (F41032). M. W. and S. S. acknowledge the support from the IAEA under Research Contract 20454 and 20447, respectively.

Data Availability Statement This manuscript has associated data in a data repository. [Author's comment: The NLD and $\gamma \mathrm{SF}$ are available online at http://mn.uio.no/fysikk/english/research/about/infrastructure/ ocl/nuclear-physics-research/compilation/ and has been deposited to the IAEA PSF database [10] (http://www-nds.iaea.org/PSFdatabase/). Raw data will be made available upon request.]

Open Access This article is licensed under a Creative Commons Attribution 4.0 International License, which permits use, sharing, adaptation, distribution and reproduction in any medium or format, as long as you give appropriate credit to the original author(s) and the source, provide a link to the Creative Commons licence, and indicate if changes were made. The images or other third party material in this article are included in the article's Creative Commons licence, unless indicated otherwise in a credit line to the material. If material is not included in the article's Creative Commons licence and your intended use is not permitted by statutory regulation or exceeds the permitted use, you will need to obtain permission directly from the copy- right holder. To view a copy of this licence, visit http://creativecomm ons.org/licenses/by/4.0/.

\section{References}

1. H.A. Bethe, An attempt to calculate the number of energy levels of a heavy nucleus. Phys. Rev. 50, 332-341 (1936). https://doi.org/10. 1103/PhysRev.50.332. https://link.aps.org/doi/10.1103/PhysRev. 50.332

2. G.A. Bartholomew, E.D. Earle, A.J. Ferguson, J.W. Knowles, M.A. Lone, Gamma-Ray Strength Functions, pages 229-324. Springer US, Boston, MA, (1973). ISBN 978-1-4615-9044-6. https://doi. org/10.1007/978-1-4615-9044-6_4

3. T. Rauscher, N. Dauphas, I. Dillmann, C. Fröhlich, Z. Fülöp, G. Gyürky, Constraining the astrophysical origin of the p-nuclei through nuclear physics and meteoritic data. Rep. Prog. Phys., 76(6), 066201, (2013). http://stacks.iop.org/0034-4885/76/i=6/ $a=066201$

4. M. Arnould, S. Goriely, The p-process of stellar nucleosynthesis: astrophysics and nuclear physics status. Phys. Rep., 384(1), 1-84, (2003). ISSN 0370-1573. https://doi.org/10.1016/ S0370-1573(03)00242-4. http://www.sciencedirect.com/science/ article/pii/S0370157303002424

5. M. Arnould, S. Goriely, K. Takahashi, The r-process of stellar nucleosynthesis: astrophysics and nuclear physics achievements and mysteries. Phys. Rep., 450(4), 97-213, (2007). ISSN 03701573. https://doi.org/10.1016/j.physrep.2007.06.002. http://www. sciencedirect.com/science/article/pii/S0370157307002438

6. S. Goriely, Radiative neutron captures by neutron-rich nuclei and the r-process nucleosynthesis. Phys. Lett. B, 436(1), 10-18, (1998). ISSN 0370-2693. https://doi.org/10.1016/ S0370-2693(98)00907-1. http://www.sciencedirect.com/science/ article/pii/S0370269398009071

7. A.C. Larsen, A. Spyrou, S.N. Liddick, M. Guttormsen, Novel techniques for constraining neutron-capture rates relevant for r-process heavy-element nucleosynthesis, (2019). ISSN 01466410. https:// www.sciencedirect.com/science/article/pii/S0146641019300298

8. M.B. Chadwick, M. Herman, P. Obložinský, M.E. Dunn, Y. Danon, A.C. Kahler, D.L. Smith, B. Pritychenko, G. Arbanas, R. Arcilla, R. Brewer, D.A. Brown, R. Capote, A.D. Carlson, Y.S. Cho, H. Derrien, K. Guber, G.M. Hale, S. Hoblit, S. Holloway, T.D. Johnson, T. Kawano, B.C. Kiedrowski, H. Kim, S. Kunieda, N.M. Larson, L. Leal, J.P. Lestone, R.C. Little, E.A. McCutchan, R.E. MacFarlane, M. MacInnes, C.M. Mattoon, R.D. McKnight, S.F. Mughabghab, G.P.A. Nobre, G. Palmiotti, A. Palumbo, M.T. Pigni, V.G. Pronyaev, R.O. Sayer, A.A. Sonzogni, N.C. Summers, P. Talou, I.J. Thompson, A. Trkov, R.L. Vogt, S.C. van der Marck, A. Wallner, M.C. White, D. Wiarda, P.G. Young, Endf/b-vii.1 nuclear data for science and technology: cross sections, covariances, fission product yields and decay data. Nucl. Data Sheets, 112(12), 2887-2996, (2011). ISSN 0090-3752. https://doi.org/10.1016/ j.nds.2011.11.002. http://www.sciencedirect.com/science/article/ pii/S009037521100113X. Special Issue on ENDF/B-VII.1 Library

9. M.B. Chadwick, Report of the nuclear physics and related computational science $R \& D$ for advanced fuel cycles workshop, (August 2006)

10. S. Goriely, P. Dimitriou, M. Wiedeking, T. Belgya, R. Firestone, J. Kopecky, M. Krtička, V. Plujko, R. Schwengner, S. Siem, H. Utsunomiya, S. Hilaire, S. Péru, Y.S. Cho, D.M. Filipescu, N. Iwamoto, T. Kawano, V. Varlamov, R. Xu, Reference database for photon strength functions. Eur. Phys. J. A 55(10), 172 (2019). https://doi.org/10.1140/epja/i2019-12840-1 
11. A. Schiller, L. Bergholt, M. Guttormsen, E. Melby, J. Rekstad, S. Siem, Extraction of level density and $\gamma$ strength function from primary $\gamma$ spectra. Nucl. Instrum. Methods Phys. Res. Sect. A, 447(3), 498-511 (2000). https://doi.org/10.1016/ S0168-9002(99)01187-0

12. M. Guttormsen, S. Goriely, A.C. Larsen, A. Görgen, T.W. Hagen, T. Renstrøm, S. Siem, N.U.H. Syed, G. Tagliente, H.K. Toft, H. Utsunomiya, A.V. Voinov, K. Wikan, Quasicontinuum $\gamma$ decay of ${ }^{91,92} \mathbf{Z r}$ : Benchmarking indirect $(n, \gamma)$ cross section measurements for the $s$ process. Phys. Rev. C 96, 024313 (2017). https://doi.org/10.1103/PhysRevC.96.024313. https://link.aps.org/doi/10.1103/PhysRevC.96.024313

13. B.V. Kheswa, M. Wiedeking, J.A. Brown, A.C. Larsen, S. Goriely, M. Guttormsen, F.L. Bello Garrote, L.A. Bernstein, D.L. Bleuel, T.K. Eriksen, F. Giacoppo, A. Görgen, B.L. Goldblum, T.W. Hagen, P.E. Koehler, M. Klintefjord, K.L. Malatji, J.E. Midtb $\varnothing$, H.T. Nyhus, P. Papka, T. Renstrøm, S.J. Rose, E. Sahin, S. Siem, T.G. Tornyi, ${ }^{137,138,139} \mathbf{L a}(n, \gamma)$. Phys. Rev. C, 95, 045805, (2017). https://doi.org/10.1103/PhysRevC.95. 045805. https://link.aps.org/doi/10.1103/PhysRevC.95.045805

14. A.C. Larsen, M. Guttormsen, R. Schwengner, D.L. Bleuel, S. Goriely, S. Harissopulos, F.L. Bello Garrote, Y. Byun, T.K. Eriksen, F. Giacoppo, A. Görgen, T.W. Hagen, M. Klintefjord, T. Renstrøm, S.J. Rose, E. Sahin, S. Siem, T.G. Tornyi, G.M. Tveten, A.V. Voinov, M. Wiedeking, Experimentally constrained $(p, \gamma)^{89} \mathrm{Y}$ and $(n, \gamma)^{89} \mathrm{Y}$ reaction rates relevant to $p$-process nucleosynthesis. Phys. Rev. C, 93, 045810, (2016). https://doi.org/10. 1103/PhysRevC.93.045810

15. A. Spyrou, S.N. Liddick, A.C. Larsen, M. Guttormsen, K. Cooper, A.C. Dombos, D.J. Morrissey, F. Naqvi, G. Perdikakis, S.J. Quinn, T. Renstrøm, J.A. Rodriguez, A. Simon, C.S. Sumithrarachchi, R.G.T. Zegers, Novel technique for constraining $r$-process $(n, \gamma)$ reaction rates. Phys. Rev. Lett. 113, 232502 (2014). https://doi.org/ 10.1103/PhysRevLett.113.232502

16. A.J. Koning, S. Hilaire, M.C. Duijvestijn, Talys-1.0, in International Conference on Nuclear Data for Science and Technology 2007, pages 2-5, Les Ulis, France, may 2008. EDP Sciences. https://doi.org/10.1051/ndata:07767

17. Micron Semiconductor Ltd. Product cataloque. Technical report, Micron Semiconductor Ltd., (2017). http://micronsemiconductor. co.uk/wp-content/uploads/2017/01/cat.pdf

18. M. Lipoglavšek, A. Likar, M. Vencelj, T. Vidmar, R.A. Bark, E. Gueorguieva, F. Komati, J.J. Lawrie, S.M. Maliage, S.M. Mullins, S.H.T. Murray, T.M. Ramashidzha, Measuring highenergy $\gamma$-rays with Ge clover detectors. Nucl. Instrum. Methods Phys. Res. Sect. A Acceler. Spectrom. Detect. Assoc. Equipment, 557(2), 523-527, (2006). ISSN 0168-9002. https://doi.org/10. 1016/j.nima.2005.11.067. http://www.sciencedirect.com/science/ article/pii/S0168900205021935

19. O.B. Tarasov, D. Bazin, LISE++: Radioactive beam production with in-flight separators. Nucl. Instrum. Methods Phys. Res. Sect. B Beam Interact. Mater. Atoms, 266(19-20), 4657-4664, (2008). ISSN 0168583X. https://doi.org/10.1016/j.nimb.2008.05. 110. http://lise.nscl.msu.edu

20. M. Guttormsen, T.S. Tveter, L. Bergholt, F. Ingebretsen, J. Rekstad, The unfolding of continuum $\gamma$-ray spectra. Nucl. Instrum. Methods Phys. Res. Sect. A 374(3), 371-376 (1996). https://doi. org/10.1016/0168-9002(96)00197-0. ISSN 01689002

21. S. Agostinelli, J. Allison, K. Amako, J. Apostolakis, H. Araujo, P. Arce, M. Asai, D. Axen, S. Banerjee, G. Barrand, F. Behner, L. Bellagamba, J. Boudreau, L. Broglia, A. Brunengo, H. Burkhardt, S. Chauvie, J. Chuma, R. Chytracek, G. Cooperman, G. Cosmo, P. Degtyarenko, A. Dell'Acqua, G. Depaola, D. Dietrich, R. Enami, A. Feliciello, C. Ferguson, H. Fesefeldt, G. Folger, F. Foppiano, A. Forti, S. Garelli, S. Giani, R. Giannitrapani, D. Gibin, J.J. Gómez Cadenas, I. González, G. Gra- cia Abril, G. Greeniaus, W. Greiner, V. Grichine, A. Grossheim, S. Guatelli, P. Gumplinger, R. Hamatsu, K. Hashimoto, H. Hasui, A. Heikkinen, A. Howard, V. Ivanchenko, A. Johnson, F.W. Jones, J. Kallenbach, N. Kanaya, M. Kawabata, Y. Kawabata, M. Kawaguti, S. Kelner, P. Kent, A. Kimura, T. Kodama, R. Kokoulin, M. Kossov, H. Kurashige, E. Lamanna, T. Lampén, V. Lara, V. Lefebure, F. Lei, M. Liendl, W. Lockman, F. Longo, S. Magni, M. Maire, E. Medernach, K. Minamimoto, P. Mora de Freitas, Y. Morita, K. Murakami, M. Nagamatu, R. Nartallo, P. Nieminen, T. Nishimura, K. Ohtsubo, M. Okamura, S. O’Neale, Y. Oohata, K. Paech, J. Perl, A. Pfeiffer, M.G. Pia, F. Ranjard, A. Rybin, S. Sadilov, E. Di Salvo, G. Santin, T. Sasaki, N. Savvas, Y. Sawada, S. Scherer, S. Sei, V. Sirotenko, D. Smith, N. Starkov, H. Stoecker, J. Sulkimo, M. Takahata, S. Tanaka, E. Tcherniaev, E. Safai Tehrani, M. Tropeano, P. Truscott, H. Uno, L. Urban, P. Urban, M. Verderi, A. Walkden, W. Wander, H. Weber, J.P. Wellisch, T. Wenaus, D.C. Williams, D. Wright, T. Yamada, H. Yoshida, D. Zschiesche, Geant4-a simulation toolkit. Nucl. Instrum. Methods Phys. Res. Sect. A Acceler. Spectrom. Detect. Assoc. Equipment, 506(3), 250-303, (2003). ISSN 0168-9002. https://doi.org/ 10.1016/S0168-9002(03)01368-8. http://www.sciencedirect.com/ science/article/pii/S0168900203013688

22. M. Guttormsen, T. Ramsøy, J. Rekstad, The first generation of $\gamma$-rays from hot nuclei. Nucl. Instum. Methods Phys. Res. Sect. A 255(3), 518-523 (1987). https://doi.org/10.1016/ 0168-9002(87)91221-6. ISSN 01689002

23. T.D. Johnson, W.D. Kulp, Nuclear data sheets for a $=87$. Nucl. Data Sheets, 129:1-190, (2015). ISSN 0090-3752. https://doi. org/10.1016/j.nds.2015.09.001. http://www.sciencedirect.com/ science/article/pii/S0090375215000460

24. R.F. Carlton, R.R. Winters, C.H. Johnson, N.W. Hill, J.A. Harvey, Total cross section and resonance spectroscopy for $\mathrm{n}+{ }^{86} \mathrm{kr}$. Phys. Rev. C 38, 1605-1618 (1988). https://doi.org/10.1103/PhysRevC. 38.1605

25. T. Ericson, V. Strutinski, On angular distributions in compound nucleus processes. Nucl. Phys. C, 8, 284293, (1958). ISSN 00295582. https://doi.org/10.1016/ 0029-5582(58)90156-1. https://www.sciencedirect.com/science/ article/pii/0029558258901561

26. T. von Egidy, D. Bucurescu, Systematics of nuclear level density parameters. Phys. Rev. C 72, 044311 (2005). https://doi.org/10. 1103/PhysRevC.72.044311

27. A. Glibert, A.G.W. Cameron, A composite nuclear-level density formula with shell corrections. Can. J. Phys. 43, 1446-1496 (1965). https://doi.org/10.1139/p65-139

28. T. von Egidy, D. Bucurescu, Experimental energy-dependent nuclear spin distributions. Phys. Rev. C 80, 054310 (2009). https:// doi.org/10.1103/PhysRevC.80.054310

29. S. Goriely, S. Hilaire, A.J. Koning, Improved microscopic nuclear level densities within the Hartree-Fock-Bogoliubov plus combinatorial method. Phys. Rev. C 78, 064307 (2008). https://doi.org/ 10.1103/PhysRevC.78.064307

30. T. Ericson, The statistical model and nuclear level densities. Adv. Phys. 9(36), 425-511 (1960). https://doi.org/10.1080/ 00018736000101239

31. E. Erba, U. Facchini, E.S. Menichella, Statistical emission in nuclear reactions and nuclear level density. Il Nuovo Cimento 22(6), 1237-1260 (1961). https://doi.org/10.1007/BF02786895

32. A.C. Larsen, M. Guttormsen, M. Krtička, E. Běták, A. Bürger, A. Görgen, H.T. Nyhus, J. Rekstad, A. Schiller, S. Siem, H.K. Toft, G.M. Tveten, A.V. Voinov, K. Wikan, Analysis of possible systematic errors in the oslo method. Phys. Rev. C 83, 034315 (2011). https://doi.org/10.1103/PhysRevC.83.034315

33. S. Raman, B. Fogelberg, J.A. Harvey, R.L. Macklin, P.H. Stelson, A. Schröder, K.L. Kratz, Overlapping $\beta$ decay and resonance 
neutron spectroscopy of levels in ${ }^{87} \mathrm{Kr}$. Phys. Rev. C 28, 602-622 (1983). https://doi.org/10.1103/PhysRevC.28.602

34. A. Voinov, M. Guttormsen, E. Melby, J. Rekstad, A. Schiller, S. Siem, $\gamma$. Phys. Rev. C 63, 044313 (2001). https://doi.org/10.1103/ PhysRevC.63.044313

35. R. Schwengner, R. Massarczyk, G. Rusev, N. Tsoneva, D. Bemmerer, R. Beyer, R. Hannaske, A.R. Junghans, J.H. Kelley, E. Kwan, H. Lenske, M. Marta, R. Raut, K.D. Schilling, A. Tonchev, W. Tornow, A. Wagner, Pygmy dipole strength in ${ }^{86} \mathrm{kr}$ and systematics of $n=50$ isotones. Phys. Rev. C 87, 024306 (2013a). https:// doi.org/10.1103/PhysRevC.87.024306

36. R. Raut, A.P. Tonchev, G. Rusev, W. Tornow, C. Iliadis, M. Lugaro, J. Buntain, S. Goriely, J.H. Kelley, R. Schwengner, A. Banu, N. Tsoneva, Cross-section measurements of the ${ }^{86} \operatorname{Kr}(\gamma, n)$ reaction to probe the $s$-process branching at ${ }^{85} \mathrm{Kr}$. Phys. Rev. Lett. 111, 112501 (2013). https://doi.org/10.1103/PhysRevLett.111.112501

37. S. Goriely, E. Khan, M. Samyn, Microscopic HFB + GRPA predictions of dipole strength for astrophysics applications. Nucl. Phys. A, 739(3), 331-352, (2004). ISSN 0375-9474. https://doi.org/ 10.1016/j.nuclphysa.2004.04.105. http://www.sciencedirect.com/ science/article/pii/S0375947404006578

38. T. Renstrøm, H.-T. Nyhus, H. Utsunomiya, R. Schwengner, S. Goriely, A.C. Larsen, D.M. Filipescu, I. Gheorghe, L.A. Bernstein, D.L. Bleuel, T. Glodariu, A. Görgen, M. Guttormsen, T.W. Hagen, B.V. Kheswa, Y.-W. Lui, D. Negi, I.E. Ruud, T. Shima, S. Siem, K. Takahisa, O. Tesileanu, T.G. Tornyi, G.M. Tveten, M. Wiedeking, Low-energy enhancement in the $\gamma$-ray strength functions of ${ }^{73,74}$ Ge. Phys. Rev. C 93, 064302 (2016). https://doi.org/10.1103/ PhysRevC.93.064302

39. M. Guttormsen, R. Chankova, U. Agvaanluvsan, E. Algin, L.A. Bernstein, F. Ingebretsen, T. Lönnroth, S. Messelt, G.E. Mitchell, J. Rekstad, A. Schiller, S. Siem, A.C. Sunde, A. Voinov, S. Ødegård, Radiative strength functions in ${ }^{93-98}$ Mo. Phys. Rev. C 71, 044307 (2005). https://doi.org/10.1103/PhysRevC.71.044307

40. A.C. Larsen, R. Chankova, M. Guttormsen, F. Ingebretsen, S. Messelt, J. Rekstad, S. Siem, N.U.H. Syed, S.W. Ødegård, T. Lönnroth, A. Schiller, A. Voinov, Microcanonical entropies and radiative strength functions of ${ }^{50,51}$ V. Phys. Rev. C 73, 064301 (2006). https://doi.org/10.1103/PhysRevC.73.064301

41. N.U.H. Syed, A.C. Larsen, A. Bürger, M. Guttormsen, S. Harissopulos, M. Kmiecik, T. Konstantinopoulos, M. Krtička, A. Lagoyannis, T. Lönnroth, K. Mazurek, M. Norby, H.T. Nyhus, G. Perdikakis, S. Siem, A. Spyrou, Extraction of thermal and electromagnetic properties in ${ }^{45}$ Ti. Phys. Rev. C 80, 044309 (2009). https://doi.org/10.1103/PhysRevC.80.044309

42. A.C. Larsen, I.E. Ruud, A. Bürger, S. Goriely, M. Guttormsen, A. Görgen, T.W. Hagen, S. Harissopulos, H.T. Nyhus, T. Renstrøm, A. Schiller, S. Siem, G.M. Tveten, A. Voinov, M. Wiedeking, Transitional $\gamma$ strength in cd isotopes. Phys. Rev. C 87, 014319 (2013a). https://doi.org/10.1103/PhysRevC.87.014319

43. A. Simon, M. Guttormsen, A.C. Larsen, C.W. Beausang, P. Humby, J.T. Burke, R.J. Casperson, R.O. Hughes, T.J. Ross, J.M. Allmond, R. Chyzh, M. Dag, J. Koglin, E. McCleskey, M. McCleskey, S. Ota, A. Saastamoinen, First observation of low-energy $\gamma$-ray enhancement in the rare-earth region. Phys. Rev. C 93, 034303 (2016). https://doi.org/10.1103/PhysRevC.93.034303

44. B.V. Kheswa, M. Wiedeking, F. Giacoppo, S. Goriely, M. Guttormsen, A.C. Larsen, F.L. Bello Garrote, T.K. Eriksen, A. Görgen, T.W. Hagen, P.E. Koehler, M. Klintefjord, H.T. Nyhus, P. Papka, T. Renstrøm, S. Rose, E. Sahin, S. Siem, T. Tornyi, Galactic production of ${ }^{138} \mathrm{La}$ : Impact of ${ }^{138,139} \mathrm{La}$ statistical properties. Phys. Lett. B, 744, 268-272, (2015). ISSN 03702693. https://doi.org/10.1016/j.physletb.2015.03.065. http://www. sciencedirect.com/science/article/pii/S0370269315002403

45. M. Wiedeking, L.A. Bernstein, M. Krtička, D.L. Bleuel, J.M. Allmond, M.S. Basunia, J.T. Burke, P. Fallon, R.B. Firestone,
B.L. Goldblum, R. Hatarik, P.T. Lake, I.-Y. Lee, S.R. Lesher, S. Paschalis, M. Petri, L. Phair, N.D. Scielzo, Low-energy enhancement in the photon strength of ${ }^{95}$ Mo. Phys. Rev. Lett. 108, 162503 (2012). https://doi.org/10.1103/PhysRevLett.108.162503

46. A.C. Larsen, N. Blasi, A. Bracco, F. Camera, T.K. Eriksen, A. Görgen, M. Guttormsen, T.W. Hagen, S. Leoni, B. Million, H.T. Nyhus, T. Renstrøm, S.J. Rose, I.E. Ruud, S. Siem, T. Tornyi, G.M. Tveten, A.V. Voinov, M. Wiedeking, Evidence for the dipole nature of the low-energy $\gamma$ enhancement in ${ }^{56} \mathrm{Fe}$. Phys. Rev. Lett. 111, 242504 (2013b). https://doi.org/10.1103/PhysRevLett.111.242504

47. A.C. Larsen, M. Guttormsen, N. Blasi, A. Bracco, F. Camera, L. Crespo Campo, T.K. Eriksen, A. Görgen, T.W. Hagen, V.W. Ingeberg, B.V. Kheswa, S. Leoni, J.E. Midtbø, B. Million, H.T. Nyhus, T. Renstrøm, S.J. Rose, I.E. Ruud, S. Siem, T.G. Tornyi, G.M. Tveten, A.V. Voinov, M. Wiedeking, F. Zeiser, Low-energy enhancement and fluctuations of $\gamma$-ray strength functions in 56,57 fe: test of the brink-axel hypothesis. J. Phys. G Nucl. Part. Phys., 44(6), 064005, (2017). http://stacks.iop.org/0954-3899/44/ $i=6 / a=064005$

48. M.D. Jones, A.O. Macchiavelli, M. Wiedeking, L.A. Bernstein, H.L. Crawford, C.M. Campbell, R.M. Clark, M. Cromaz, P. Fallon, I.Y. Lee, M. Salathe, A. Wiens, A.D. Ayangeakaa, D.L. Bleuel, S. Bottoni, M.P. Carpenter, H.M. Davids, J. Elson, A. Görgen, M. Guttormsen, R.V.F. Janssens, J.E. Kinnison, L. Kirsch, A.C. Larsen, T. Lauritsen, W. Reviol, D.G. Sarantites, S. Siem, A.V. Voinov, S. Zhu, Examination of the low-energy enhancement of the $\gamma$-ray strength function of ${ }^{56} \mathrm{Fe}$. Phys. Rev. C 97, 024327 (2018). https:// doi.org/10.1103/PhysRevC.97.024327

49. A.C. Larsen, S. Goriely, Impact of a low-energy enhancement in the $\gamma$-ray strength function on the neutron-capture cross section. Phys. Rev. C 82, 014318 (2010). https://doi.org/10.1103/PhysRevC.82. 014318

50. R. Schwengner, S. Frauendorf, A.C. Larsen, Low-energy enhancement of magnetic dipole radiation. Phys. Rev. Lett. 111, 232504 (2013b). https://doi.org/10.1103/PhysRevLett.111.232504

51. M. Czerwiński, T. Rząca-Urban, W. Urban, P. Bączyk, K. Sieja, B.M. Nyakó, J. Timár, I. Kuti, T.G. Tornyi, L. Atanasova, A. Blanc, M. Jentschel, P. Mutti, U. Köster, T. Soldner, G. de France, G.S. Simpson, C.A. Ur, Neutron-proton multiplets in the nucleus ${ }^{88}$ Br. Phys. Rev. C 92, 014328 (2015). https://doi.org/10.1103/ PhysRevC.92.014328

52. J. Litzinger, A. Blazhev, A. Dewald, F. Didierjean, G. Duchêne, C. Fransen, R. Lozeva, K. Sieja, D. Verney, G. de Angelis, D. Bazzacco, B. Birkenbach, S. Bottoni, A. Bracco, T. Braunroth, B. Cederwall, L. Corradi, F .C .L. Crespi, P. Désesquelles, J. Eberth, E. Ellinger, E. Farnea, E. Fioretto, R. Gernhäuser, A. Goasduff, A. Görgen, A. Gottardo, J. Grebosz, M. Hackstein, H. Hess, F. Ibrahim, J. Jolie, A. Jungclaus, K. Kolos, W. Korten, S. Leoni, S. Lunardi, A. Maj, R. Menegazzo, D. Mengoni, C. Michelagnoli, T. Mijatovic, B. Million, O. Möller, V. Modamio, G. Montagnoli, D. Montanari, A .I. Morales, D .R. Napoli, M. Niikura, G. Pollarolo, A. Pullia, B. Quintana, F. Recchia, P. Reiter, D. Rosso, E. Sahin, M.D. Salsac, F. Scarlassara, P.-A. Söderström, A .M. Stefanini, O. Stezowski, S. Szilner, Ch. Theisen, J .J. Valiente Dobón, V. Vandone, A. Vogt, Transition probabilities in neutron-rich ${ }^{84,86} \mathrm{Se}$. Phys. Rev. C 92, 064322 (2015). https://doi.org/10.1103/PhysRevC.92. 064322

53. E. Caurier, G. Martínez-Pinedo, F. Nowacki, A. Poves, A.P. Zuker, The shell model as a unified view of nuclear structure. Rev. Mod. Phys. 77, 427-488 (2005). https://doi.org/10.1103/RevModPhys. 77.427

54. K. Sieja, Electric and magnetic dipole strength at low energy. Phys. Rev. Lett. 119, 052502 (2017). https://doi.org/10.1103/ PhysRevLett.119.052502

55. A.J. Koning, J.P. Delaroche, Local and global nucleon optical models from $1 \mathrm{kev}$ to 200 mev. Nucl. Phys. A, 713(3), 
231-310, (2003). ISSN 0375-9474. https://doi.org/10.1016/ S0375-9474(02)01321-0. http://www.sciencedirect.com/science/ article/pii/S0375947402013210

56. W. Hauser, H. Feshbach, The inelastic scattering of neutrons. Phys. Rev. 87, 366-373 (1952). https://doi.org/10.1103/PhysRev.87.366

57. E. Bauge, J.P. Delaroche, M. Girod, Lane-consistent, semimicroscopic nucleon-nucleus optical model. Phys. Rev. C 63, 024607 (2001). https://doi.org/10.1103/PhysRevC.63.024607

58. M. Bhike, E. Rubino, M.E. Gooden, Krishichayan, W. Tornow, Measurements of the ${ }^{86} \mathrm{Kr}(n, \gamma){ }^{87} \mathrm{Kr}$ and ${ }^{86} \mathrm{Kr}(n, 2 n)^{85} \mathrm{Kr}^{m}$ reaction cross sections below $E_{n}=15$ mev. Phys. Rev. C 92, 014624 (2015). https://doi.org/10.1103/PhysRevC.92.014624

59. G. Walter, B. Leugers, F. Käppeler, Z.Y. Bao, G. Reffo, F. Fabbri, Kilo-electron-volt neutron capture cross sections of the krypton isotopes. Nucl. Sci. Eng. 93, 357-369 (1986). https://doi.org/10. 13182/NSE86-A18471

60. H. Beer, P.V. Sedyshev, W. Rochow, P. Mohr, H. Oberhummer, Neutron capture measurements of the noble gas isotopes $22 \mathrm{Ne}$, $40 \mathrm{Ar}$ and $78,80,84,86 \mathrm{Kr}$ in the $\mathrm{keV}$ energy region. Nucl. Phys. A, 705(1-2), 239-261, (2002). ISSN 03759474. https://doi. org/10.1016/S0375-9474(02)00645-0. https://www.sciencedirect. com/science/article/abs/pii/S0375947402006450

61. I. Dillmann, Kadonis - the karlsruhe astrophysical database of nucleosynthesis in stars. In: AIP conference proceedings (2006). https://doi.org/10.1063/1.2187846. http://www.kadonis.org
62. T. Rauscher, F.K. Thielemann, K.L. Kratz, Nuclear level density and the determination of thermonuclear rates for astrophysics. Phys. Rev. C Nucl. Phys. 56(3), 1613-1625 (1997). https://doi. org/10.1103/PhysRevC.56.1613. ISSN 1089490X

63. D. Rochman, A.J. Koning, J. Kopecky, J.C. Sublet, P. Ribon, M. Moxon, From average parameters to statistical resolved resonances. Ann. Nucl. Energy, 51, 60-68, (2013). ISSN 03064549. https://doi.org/10.1016/j.anucene.2012.08.015. https:// www.sciencedirect.com/science/article/pii/S0306454912003350

64. D. Rochman, S. Goriely, A.J. Koning, H. Ferroukhi, Radiative neutron capture: Hauser Feshbach vs. statistical resonances. Phys. Lett. B 764, 109-113 (2017). https://doi.org/10.1016/j.physletb.2016. 11.018

65. M. Krtička, M. Wiedeking, F. Bečvář, S. Valenta, Consistency of photon strength function models with data from the ${ }^{94} \mathrm{Mo}(\mathrm{d}, \mathrm{p} \gamma \gamma)$ reaction. Phys. Rev. C 93, 054311 (2016). https://doi.org/10.1103/ PhysRevC.93.054311 